Formiga, N. S., \& Borges, L. P. (2021). Evidência Empírica do Inventário sobre Desordem da Dismorfia Muscular em Praticantes Regulares de Academia. Consumer Behavior Review, 5(3), 466-483.

DOI: https://doi.org/10.51359/2526-7884.2021.249161

ISSN: 2526-7884

Editor: Prof. Dr. Marconi Freitas da Costa

E-mail: cbr@ufpe.br
Avaliação: Double blind review

Recebido: 27 de dezembro de 2020

Aprovado: 02 de junho de 2021

\title{
EVIDÊNCIA EMPÍRICA DO INVENTÁRIO SOBRE DESORDEM DA DISMORFIA MUSCULAR EM PRATICANTES REGULARES DE ACADEMIA
}

\section{Empirical evidence from the Muscle dysmorphic disorder inventory in regular gym practitioners}

\author{
Nilton Soares Formiga ${ }^{1}$ \\ ORCID: http://orcid.org/0000-0003-4907-9736 \\ E-mail:nsformiga@yahoo.com \\ Lorena Pimentel Borges ${ }^{1}$ \\ ORCID: https://orcid.org/0000-0003-4511-491X \\ E-mail: lorenaborgespimentel@hotmail.com
}

${ }^{1}$ Universidade Potiguar, Natal, Brasil

\begin{abstract}
Resumo
O objetivo central deste trabalho é traduzir, adaptar e validar o The Muscle Dysmorphic Disorder Inventory - MDDI (Inventário de Transtorno de Dismorfia Muscular) em praticantes regulares da academia. Participaram do estudo 170 sujeitos, homens e mulheres acima de 18 anos, respondendo a um questionário com itens referentes aos dados sociodemográficos, sobre características da atividade física na academia e a escala MDDI. 0 questionário foi aplicado aos participantes de forma individual, através de um formulário eletrônico hospedado no google.forms. Observou-se que a MDDI apresentou uma fatorialização semelhante à original, com os seus três fatores interrelacionados. Em relação
\end{abstract}

\begin{abstract}
The main goal of this paper is to translate, adapt and validate The Muscle Dysmorphic Disorder Inventory - MDDI in regular practitioners at the gym. The study included 170 subjects between men and women over 18 years old; they answer a questionnaire about sociodemographic data, on physical activity in the gym and about MDDI scale. The questionnaire was applied to participants individually, through an electronic form hosted on google.forms. It was observed that the MDDI presented a factorization similar to the original, with its three factors that interact with each other. Regarding to the convergence and divergence validity, both MDDI, considered a single factor, and its
\end{abstract}


a validade de convergência e divergência, tanto o MDDI, considerado um fator único, quanto seus fatores (Desejo por tamanho do corpo; Intolerância à Aparência e Comprometimento Funcional) relacionaram-se com a autoestima positiva e negativa, tendo os escores correlacionais sido maiores com a autoestima negativa. Não apenas corroborou a escala MDDI e sua tri-fatorialidade, a qual se assemelhou à versão americana e alemã da mesma escala, bem como este construto tem uma influência em variáveis psicológicas, no caso, da autoestima. Sendo assim, a escala seria útil quanto avaliação e desenvolvimento de práticas com acompanhamento para uma saúde do exercício.

Palavras-chave: Dismorfia Muscular; Vigorexia; Atividades físicas. factors (DT $=$ Desire for body size; $\mathrm{IA}=$ Appearance Intolerance; $\mathrm{CF}=$ Functional Impairment) were related to positive and negative self-esteem; however, correlational scores were higher with the negative selfesteem. It did not only corroborated the MDDI scale and its three-factor, which resemble to the American version and the German version of the same scale, but this construct also has an influence over psychological variables, in this case, the self-esteem. Thus, the scale would be useful in terms of assessing and developing practices with monitoring for exercise health.

Keywords: Muscular Dysmorphia; Vigorexia; Physical activities.

\section{INTRODUÇÃO}

Apesar do corpo, geralmente, ser visto como, a partir das concepções da ciência da saúde, como um organismo bastante complexo pelas suas características fisiológicas em sua estrutura e função. É por meio dele que as experiências ocorrem, mediado pela sua existência, além de ser moldado pelo processo cultural de cada sociedade gerindo uma construção social que é capaz de influenciar o sujeito e o desenvolvimento psíquico sobre o si mesmo e a determinação do que é e como é o seu corpo (cf. Mendes \& Nóbrega, 2004; Santos et al., 2013)

Para Petroski, Pelegrini e Glaner (2010), a imagem do corpo pode ser considerada como uma construção multidimensional, pois representam como os indivíduos pensam, sentem e agem a respeito de seus atributos físicos. Todavia, tem-se notado que nas sociedades contemporâneas vêm demonstrando uma preocupação bastante exagerada não apenas sobre sentido e o e significado do corpo, mas, também, uma espécie de 'enquadramento', mas, dos padrões de beleza e estética, do que de fato do corpo em si (Gonçalves, 2014).

Desta forma, percebe-se uma referência de beleza, a qual, atende menos a realidade humano com seu corpo e mais a realidade imagética-midiática ideal de corpo. Esta, tem ultrapassado os limites geopolíticos e culturais, pois, com o alcance da mídia em geral, atinge diretamente a população, independente da condição social e econômica, ocasionando uma insatisfação com a imagem corporal real e, muitas vezes, recorrendo a dietas e mudanças de comportamento fora dos padrões de normalidade (Petroski et al., 2010; Forbes, Jung, Vaamonde, Omar, Paris, \& Formiga, 2012).

Segundo Severiano et al. (2010; cf. Lipovetsky, 1989, 2007; Gomes, 2017; Jesus et al., 2016), a indústria da beleza, por meio de vários meios de comunicação de massa (Instagram, facebook, blogs, revistas, televisão), tem sugerido padrões de beleza e, consequentemente, expõem as pessoas e seus corpos a uma série de restrições e modelos ideais, tudo em busca de um suposto "bem-estar" e felicidade para uma vida "melhor".

Assim, se as pessoas divergem em concepção e realidade ao padrão considerado ideal pela mídia, elas desenvolvem uma certa percepção de exclusão, fracasso, podendo, com isso, ocasionar um sofrimento psíquico (Bittencourt, 2013). Torna-se comum que as demandas psicológicas sejam codependentes do contexto social da concepção de beleza existida, ou seja, diante da pressão exercida, as pessoas se apresentem com certo desajuste psicológico, justamente, por não ser possível atender a um princípio do ideal (Moré et al., 2008; Marques, Legal, \& Höfelmann, 2012). 
Assunção (2002) cita que a preocupação com o corpo sempre foi algo quase que exclusivo das mulheres, porém, nos últimos tempos tem-se visto que homens e mulheres vêm evidenciando essa preocupação igualitariamente. Wade (2000) refere-se que quando os homens se veem diante de outros homens com características físicas atraentes, demonstram sentir uma insatisfação com o seu próprio corpo, parecendo afetar consideravelmente sua autoavaliação e muitas vezes, sua autoestima.

Sendo assim, provavelmente, à atenção com a saúde e a forma corporal faz com que demandas na área de psicologia venham surgir. Assim, o crescente aumento no nível de preocupação com a aparência, o culto ao corpo e a intensa valorização da imagem, desencadeiam uma série de problemas advindos da nova ordem da sociedade atual.

Dessa forma, nota-se que algumas dessas pessoas muitas vezes apresentam alguns distúrbios na percepção corporal, como é o caso da Dismorfia Muscular, conhecida também como Vigorexia (Utiyama, 2011; cf. Vasconcelos, 2013). Fenômeno este, que vem se apresentando como algo comum no mundo contemporâneo (Neumark-Sztainer, Paxton, Hannan, Haines, \& Story, 2006; Azevedo, Ferreira, Silva, Caminha, \& Freitas, 2012; Motter, Almeida, \& Bellini, 2017).

De acordo com o a quinta edição do Manual Diagnóstico e Estatístico de Transtornos Mentais (cf. Associação Americana de Psiquiatria, 2013), a dismorfia muscular é "uma forma de transtorno dismórfico corporal caracterizado pela crença de que a estrutura corporal do indivíduo é muito pequena e insuficientemente musculosa" (p. 242). Trata-se, especificamente, de um distúrbio na imagem corporal semelhante ao observado na anorexia nervosa, porém, os indivíduos com anorexia se percebem como mais gordos do que realmente são, enquanto aqueles que apresentam dismorfia muscular se veem como menores ou mais fracos (Olivardia, 2001; cf. Fortes et al., 2017).

De acordo com Severiano et al. (2010), Dismorfia Muscular se caracteriza por ser um transtorno no qual as pessoas praticam atividades esportivas intensamente e continuamente, com objetivos de ganhar massa muscular, sem se preocupar com as consequências de seus atos, com a sua saúde ou com qualquer contraindicação.

Assim, a vigorexia se apresenta com traços narcísicos, pois abusa do culto ao corpo e recorre a qualquer recurso para atingir o corpo perfeito (Severiano et al., 2010). As preocupações com o corpo podem causar ansiedade significativa, os pensamentos são muito intrusivos, interferem na concentração, e consomem excessivo tempo e energia do indivíduo, assim como sentir-se gordo pode ser devastador para alguém que sofre de anorexia nervosa (Olivardia, 2001).

A dismorfia muscular está associada a uma série de outros distúrbios psiquiátricos, como por exemplo, distúrbios alimentares, distúrbios de humor e ansiedade (Pope, Gruber, Choi, Olivardia, \& Phillips, 1997) e indivíduos com dismorfia muscular raramente procuram tratamento (Olivardia, 2001). Frente a tais reflexões e levando em consideração o crescente número de estudos relacionados à dismorfia muscular, em consulta realizada nas bases de dados da publicação científica no Brasil, ainda é escassa a literatura nacional, tanto sobre esta temática em frequentadores de academias quanto, uma medida consistência e fidedigna que fosse capaz de mensurar esse fenômeno nas pessoas. Ambos os aspectos contribuiriam para à avaliação na população brasileira ou até amostras bastantes especificas, os índices, bem como, critérios de diagnóstico e descrição deste transtorno nas mais distintas amostras.

Com isso, o presente artigo tem como objetivo a tradução, adaptação e verificação fatorial e validação do construto o Transtorno de Dismorfia Muscular para contexto brasileiro. Trata-se de um inventário americano que representa uma nova ferramenta de triagem para a avaliação e diagnóstico deste transtorno, conhecido como The Muscle Dysmorphic Disorder Inventory - MDDI, o qual, foi desenvolvido por Schlundt, Woodford e Brownlee (2000) e validade para a língua inglesa por Hildebrandt et al. (2004).

\section{MÉTODO}

\section{Amostra}

Tratar-se-á de uma pesquisa quantitativa, do tipo descritivo, exploratório e correlacional, envolvendo pessoas que fazem atividades físicas, regularmente, em academias. 
A amostra referente foi verificada através do pacote estatístico G Power 3.1, software, o qual, é utilizado para calcular o poder estatístico relacionando o ' $n$ ' necessário para a pesquisa e tipo de cálculo a ser realizado (Faul, Erdfelder, Lang, \& Buchner, 2007).

A coleta de dados realizou-se através do método bola de neve, pois, considerou-se a disponibilidade e interesse do respondente em participar da pesquisa. Para isso, considerar-se-á uma probabilidade de 95\% ( $\mathrm{p}<0,05)$, magnitude do efeito amostral $(\mathrm{r} \geq 0,50)$ e um padrão de poder hipotético $(\pi \geq 0,80)$ destinada verificação da qualidade e significância da amostra para a realização da pesquisa.

Sendo assim, uma amostra final de 170 respondentes revelou-se ser suficiente para a realização dessa pesquisa, para a qual, observaram-se os seguintes indicadores estatísticos $(\mathrm{t} \geq 1,98 ; \pi$ $\geq 0,96, p<0,05$ ) que correspondiam a segurança e significância da amostra.

Quanto ao critério de inclusão considerou-se: ter idade entre 18 e 50 anos de idade, homens e mulheres, serem alfabetizados, residirem no Rio Grande do Norte e frequentarem academias para a prática de exercícios físicos pelos menos uma vez durante a semana, durante uma hora de atividade.

No que se refere aos critérios de Exclusão, estabeleceram-se os seguintes: negar-se assinar o termo de consentimento livre e esclarecido, não completar as respostas de todo o instrumento, ter idade abaixo de 18 anos, não ser alfabetizado, não residir no Rio Grande do Norte e não fazer nenhuma atividade física em academia.

\section{Instrumentos}

Os participantes responderam aos seguintes instrumentos:

The Muscle Dysmorphic Disorder Inventory - MDDI (Inventário de desordem de disformia muscular). Trata-se de um instrumento desenvolvimento por Schlundt, Woodford e Brownlee (2000), compost por 16 itens de autorrelato, com base nos critérios de pesquisa do MD, mas não incluiu nenhuma pergunta que avaliasse a característica de comprometimento funcional do DM. Para aprimorar essa medida e criar uma avaliação multidimensional do DM que avaliou características cognitivas, comportamentais e emocionais, três itens foram eliminados e sete foram adicionados. Os 21 itens finais incluíram sete perguntas que avaliaram os três fatores de diagnóstico associados à DM desejo por tamanho, ansiedade e esquiva de aparência e comprometimento funcional. Os participantes classificam todas as perguntas em uma escala do tipo Likert de 5 pontos, de "nunca" a "sempre". As dimensões MDDI incluem perguntas referentes a cognições, emoções e comportamentos relacionados à imagem corporal.

A subescala do desejo por tamanho (DFS) consiste em perguntas sobre pensamentos de ser menor, menos musculoso e mais fraco que o desejado, ou deseja aumentar o tamanho e a força. 0 estilo de pensamento representado por essa subescala é consistente com as preocupações sobre tamanho inadequado no DM, conforme descrito por Pope et al. (1997). A subescala Intolerância à aparência (AI) consiste em perguntas sobre crenças negativas sobre o corpo e a ansiedade resultante da aparência ou prevenção da exposição corporal. As cognições negativas e as manifestações comportamentais das crenças negativas essenciais sobre o corpo de alguém são consistentes com as características do DM, como vestir roupas largas na praia ou crer que o corpo é feio e desagradável. Por fim, a subescala de comprometimento funcional (FI) consiste em perguntas sobre comportamentos relacionados à manutenção de rotinas de exercícios, interferência de emoções negativas ao se desviar das rotinas de exercícios ou prevenção de situações sociais devido a sentimentos negativos e preocupação com o corpo

Zeeck, Welter, Alatas, Hildebrandt, Lahmann e Hartmann (2018), validaram o MDDI para o contexto alemão; estes autores utilizaram uma medida reduzida, com 13 itens, observando que não apenas revelou indicadores psicométricos consistentes, com a existência das três dimensões proposta pelos autores originais, bem como, quanto validade convergente, o MDDI relacionou-se positivamente, com a dependência do exercício, mas, por outro lado, houve uma correlação negativa entre a intolerância à aparência e a aceitação do próprio corpo.

Para a versão brasileira, a escala foi traduzida por uma especialista na língua inglesa e, novamente, retraduzida, buscando verificar a qualidade semântica dos itens. Desta maneira, a escala composta por 13 itens (MDDI1 = A minha vontade é ficar mais forte, bem malhado e definido; MDDI2 = 
Acho que meu corpo é muito pequeno, não tenho músculos o bastante, isto é, não sou "fortão"; MDDI3 = Acho que meu peitoral é muito pequeno não tem músculos tão bem definidos, do tipo malhadão; MDDI4 = Minha vontade é que meus braços fossem maiores, com músculos mais definidos e malhados; MDDI5 = Acho que minhas pernas são finas demais e pouco definidas na musculatura; MDDI6 = Eu sou muito tímido quando se trata de deixar as pessoas me verem sem camisa; MDDI7 = Eu uso roupas largas para que as pessoas não possam ver meu corpo; MDDI8 = Eu sinto que tenho muita gordura corporal; MDDI9 = Eu odeio meu corpo; MDDI10 = Já desperdicei chances para conhecer pessoas por causa dos meus horários para ir a academia (fazer os exercícios físicos); MDDI11 = Sinto-me deprimido quando falto um ou mais dias do meu treino de exercícios físicos; MDDI12 = Eu deixo de aproveitar atividades sociais (por exemplo, assistir a jogos de futebol, jantar, ir ao cinema, etc.) com amigos por causa da minha agenda de exercícios físicos e MDDI13 = Sinto-me ansioso quando falto um ou mais dias do meu treino de exercícios físicos) foi administrada aos participantes, os quais, frequentadores de academias e que se auto-percebem preocupados de forma exagerada com o corpo e sua estrutura muscular, caracterizando-se como dismorfia muscular.

Para mensurar o construto, o respondente deveria indicar no espaço relativo a sua resposta, apresentado no instrumento, um círculo ou uma marcação na cor escura, em uma escala de cinco pontos que variava de $0=$ nunca a $4=$ sempre a respeito do que cada questão expressava.

Escala de autoestima: Desenvolvida por Rosenberg (1965) (Rosenberg self-esteem scale) e foi selecionada para este estudo mediante um levantamento bibliográfico em bases de dados nacionais e internacionais sobre instrumentos que avaliam esta variável. Para a sua escolha levamos em consideração algumas questões consideradas relevantes: a faixa etária da amostra original do estudo é compatível a este. 0 processo de criação foi realizado com um grande contingente (5024) de estudantes pertencentes às escolas públicas; a escala original possui bons coeficientes psicométricos (Alfa de Cronbach de 0.92) e este padrão de coeficiente é observado nos trabalhos de Hutz (2000; Giacomoni, 2002). Essa escala se organiza da seguinte forma: autoestima positiva (AE 1 - Sinto que não sou uma pessoa que vale alguma coisa, AE3 - Em geral penso que sou um fracasso, AE4 - Sou incapaz de fazer as coisas tão bem quanto os demais, AE7 - Em geral estou insatisfeito comigo mesmo, AE10 Às vezes penso que sou bom para alguma coisa) e autoestima negativa (AE2 - Sinto que não tenho boas qualidades, AE5 - Sinto que não tenho com o que estar orgulhoso, AE6 - Tenho uma atitude negativa comigo mesmo, AE8 - Eu tenho motivos para me orgulhar da vida, AE9 - Às vezes me sinto inútil).

Escala de autoestima pode ser avaliada segundo os níveis: baixo, médio e alto. A baixa autoestima se caracteriza pelo sentimento de incompetência, de inadequação à vida e incapacidade de superação de desafios; a alta expressa um sentimento de confiança e competência; e a média flutua entre o sentimento de adequação ou inadequação, manifestando essa inconsistência no comportamento (Branden, 2000; Rosenberg, 1973).

Tolentino et al. (2016), verificaram a qualidade da estrtura fatorial desta medida e de acordo com os resultados obtidos, esta escala revelou indicadores estatísticos que justificam a sua consistência fatorial, confirmando os dois fatores: autoestima negativa e autoestima positiva.

Além dessas escalas, havia um questionário sociodemográfico (aspectos relacionados a sexo, idade, renda, nível educacional) e de atividade física (aspectos relacionados a variáveis consideradas importantes para o melhor entendimento da vigorexia).

\section{Administração da pesquisa e análise de dados}

Todos os procedimentos adotados, nesta pesquisa, seguiram as orientações previstas na Resolução 466/2012 do CNS e na Resolução 016/2000 do Conselho Federal de Psicologia para as pesquisas com seres humanos (Conselho Nacional de Saúde [CNS], 2012; Associação Nacional de Pesquisa e Pós-Graduação em Psicologia [ANPEPP], 2000).

Após a submissão e aprovação do problema realizada pelo conselho de ética da Plataforma Brasil submetido ao CONEP sob o protocolo de pesquisa CAAE no 24995619.7.0000.5296, o instrumento da pesquisa foi aplicado as mães de forma individual, através de um formulário eletrônico hospedado no google.docs. De acordo com a disponibilidade do tempo e espaço físico dos 
respondentes em seus setores de trabalho ou fora dele, ele será convidado a participar de forma voluntária, anônima e privada em responder o questionário.

Para isso, foi encaminhado para o e-mail, telefone particular (através das redes sociais que eles pudessem fazer parte) e nas redes sociais. Será informado aos que desejavam participar, quando acessaram o endereço da pesquisa, que a pesquisa será anônima e sigilosa, serão apresentadas as principais informações acerca do objetivo da pesquisa, as instruções para entendimento das questões, bem como, que o participante pode desistir a qualquer momento da pesquisa sem prejuízo algum para ele.

O participante que concordar em participar da pesquisa, por vontade própria, assentindo com o objetivo do estudo, bem como, os riscos e benefícios, passará ao próximo passo do estudo, que será responder o questionário.

Tendo em vista que os objetivos deste estudo são a tradução e adaptação do inventário MDDI, serão realizados os seguintes passos para: a) tradução da versão original do inventário em inglês para o português feito por dois pesquisadores brasileiros; b) comparação das duas traduções e, a partir da síntese final, a realização de uma terceira versão; c) tradução reversa. Todo esse processo resultará na versão brasileira do inventário.

Em relação a análise dos dados, utilizar-se-á o pacote estatístico SPSSWIN, em sua versão 22.0, para tabular os dados e realizar as análises estatísticas descritivas e os cálculos referentes correlação de Pearson, teste $t$ de Student, alfa de Cronbach e qui-quadrado.

No mesmo pacote estatístico, realizou-se a análise de Componentes Principais (CP), tomando como critérios para decisão fatorial, o KMO igual ou superior a 0,70 e o Teste de Esfericidade de Bartlett (qui-quadrado, $\chi^{2}$ ) significativo $(p<0,05)$ (Tabachnick \& Fidell, 2001; Dancey \& Reidy, 2006); considerou-se ainda, os critérios de Kaiser (valor próprio igual ou superior a 1) e Cattell (distribuição gráfica dos valores próprios, visando distinguir aqueles sobressalentes) e análise Paralela (Dancey \& Reidy, 2006; Ledesma \& Valero-Mora, 2007).

\section{Análise de riscos e benefícios}

Os riscos que a pesquisa pode apresentar são mínimos, pois os procedimentos a serem realizados se fundamentarão no sigilo e na ética, e não possuem intuito de emergir emoções negativas ou desconfortáveis para os participantes, como desconforto ou constrangimento. Porém, tendo em vista que tais riscos podem ocorrer, os mesmos podem ser amenizados através do anonimato, bem como, o questionário será aplicado por meio eletrônico através do Google forms, de maneira a minimizar a exposição dos participantes dessa pesquisa.

Não haverá nenhum benefício direto ao participante, entretanto, a partir dos estudos realizados nesta pesquisa, procurar-se-á contribuir para a prática profissional da saúde no diagnóstico da dismorfia muscular, bem como, a partir disso, oferecer subsídio ao planejamento de intervenções com a população acometida por este problema e intervenções preventivas.

\section{RESULTADOS}

Finalizada a coleta de dados e tendo inserido as respostas dos sujeitos no programa Excell, de acordo com o que era apresentado nos instrumentos da pesquisa no site em que foi hospedado. Realizou-se em seguida uma inspeção visual quanto aos valores máximos e mínimos das escalas das respostas. Nesta etapa, buscou-se atender aos pressupostos dos cálculos de representatividade e fidedignidade das medidas utilizadas, verificando se não teriam respostas duplicadas e/ou missing (itens sem respostas).

Considerando que a medida utilizada foi desenvolvida por Schlundt, Woodford e Brownlee (2000) e, posteriormente, por Hildebranch et al (2004) em outro contexto social, considera-se que ela apresenta uma originalidade para o contexto da análise do construto no contexto brasileiro. Para isso, procurou-se, atender alguns critérios de análise qualitativa (tradução e re-tradução) e quantitativa (análise de conteúdo e semântica).

Esta condição se deve as seguintes direções: 1 - por ser uma pesquisa pioneira com frequentadores de academia para desenvolvimento físico e muscular), 2 - devido as recentes buscas 
nos sites da produção científica brasileira na área da psicologia geral, psicologia do consumo, entre outras áreas, durante o período de Abril de 2019 a Março de 2020 (por exemplo, scielo.br, newpsi.bvspsi.org.br, pepsic.bvsalud.org) não foram encontrados estudos sobre o tema tratado neste artigo.

Buscando atender aos objetivos pretendidos, efetuaram-se análises sobre a qualidade da amostra. Assim, em relação aos dados omissos das pesquisas, observou-se que esteve abaixo de 5\%, com isso, é possível substituídos pela média ou moda dos dados da amostra, no caso de encontrá-los no banco de dados. Em relação a multicolinearidade entre as variáveis, as correlações entre elas estiveram dentro dos parâmetros definidos por Tabachnick e Fidell $(2001)$ [ $r \leq 0,90$, variando de $-0,08$ a 0,43$]$.

Tais condições expressas no parágrafo acima, revela a não existência de variáveis com muita correlação, condição que permite a elaboração de modelos correlacionais com baixo erro de medida. Verificou-se também, a presença de outliers multivariados, realizado através do teste de normalidade de Kolmogorov-Smirnov (KS), o qual, se destina a análise de amostras superiores a 100 sujeitos (cf. Nascimento, Tibana, Fereira, \& Prestes, 2014; Miot, 2017), observou-se uma normalidade $(\mathrm{KS}=0,68$ ) da amostra a um $\mathrm{p}<0,37$.

Verificou-se, também, as diferenças em assimetria através dos histogramas com superposição da curva normal e dos índices de assimetria. Tendo com orientação os parâmetros definidos por Miles e Shevlin (2001), os quais, afirmam que, ainda que os dados da amostra não se apresentem com uma distribuição normal, sendo o número estatístico de assimetria menor que 1,0, haverá pouco problema, e se ele se apresentar entre 1,0 e 2,0, ainda assim, é considerado aceitável. Desta forma, ao avaliar os índices de assimetria das medidas, todos os valores da assimetria encontrados permaneceram dentro dos parâmetros aceitáveis, os quais variaram de -0,58 a 1,77.

É necessário destacar que, tanto as variáveis independentes (VI) quanto as dependentes (VD) mensuram opiniões dos mesmos respondentes (funcionários públicos do mesmo munícipio, no qual, a pesquisa foi realizada) podendo encontrar uma variância comum do método (VCM) (common method variance) representando, com isso, um problema; para isso, empregou-se o teste de um fator de Harman para examinar a existência de algum viés (variância) de método comum (VCM) nos dados coletados, conforme sugestão de Podsakoff, MacKenzie e Podsakoff (2003).

Observou-se que tanto para a amostra em questão foi encontrado um único fator com autovalor acima de 1,00, o que explicou um valor menor do que $50 \%$, observando que houve um percentual de $27,82 \%$ da covariância nas variáveis, o que implica a inexistência de um problema de variância comum do método.

Além disso, testou-se também a tendência de não resposta, usando o procedimento de Armstrong e Overton (1977), através do qual se avaliou as diferenças entre os primeiros (n1=10) e os últimos $(\mathrm{n} 2=10)$ participantes da pesquisa; para isso, utilizou-se o teste de Levene para verificar a igualdade das variâncias e um teste $t$ para avaliar a igualdade dos meios. Sendo assim considerado, observou-se que não houve resultado significativo a um nível de $5 \%$ entre os escores médios dos constructos (com $t \leq 1,96$ ), o que revela o viés de não resposta, condição esta que não representa ser um problema significativo.

Confirmado a qualidade da amostra, procurou-se atender aos objetivos principais do artigs. Para isso, algumas etapas avaliativas, estabelecidas como hierarquia qualitativa para a construção e definição do instrumento proposto, foram seguidas, considerados tipo de estudos.

Antes de seguir para essas etapas, apresentar-se-á as informações sociodemográficas da amostra coletada: foram 170 respondentes, todos frequentadores de academia na cidade de Natal-RN, com idades variando 18 a 56 anos (Media $=31,04$, d.p. $=10,44$ ), a maioria $(75 \%$ ) eram mulheres, 71\% deles trabalham, sendo $74 \%$ do setor privado, $67 \%$ tem curso superior e com $48 \%$ tendo uma renda familiar acima de 4.500,00 reais. $67 \%$ dos respondentes quando vão a academia, frequentam a entre uma e duas horas e $72 \%$ de dois a três dias.

\section{Estudo 1: Tradução do instrumento MDDI}

Por ser um instrumento original em língua inglesa; este, passou por uma tradução com especialistas, os quais, fluentes no inglês. Assim, dois profissionais, na área da Psicologia e com 
domínio no inglês, realizou a tradução do instrumento. Na tabela 1, é possível observar que a tradução do instrumento foi viável e revelou baixo distanciamento no sentido e significado do instrumento original para a sua adaptação na língua do português brasileiro.

Tabela 1

Tradução da escala MDDI

\begin{tabular}{|c|c|}
\hline Original & Tradução português \\
\hline Drive for size & Desejo por tamanho do corpo \\
\hline 1. I wish I could get bigger & $\begin{array}{l}\text { 1. A minha vontade é de ficar mais forte, bem } \\
\text { malhado e definido. }\end{array}$ \\
\hline 2. I think my body is too small & $\begin{array}{l}\text { 2. Acho que não tenho músculos o bastante, isto é, } \\
\text { não sou 'fortão'. }\end{array}$ \\
\hline 3. I think my chest is too small & $\begin{array}{l}\text { 3. Acho que meu peitoral não tem músculos tão bem } \\
\text { definidos, do tipo malhadão. }\end{array}$ \\
\hline 4. I wish my arms were bigger & $\begin{array}{l}\text { 4. Minha vontade é que meus braços tivessem } \\
\text { músculos mais definidos e malhados. }\end{array}$ \\
\hline 5. I think my legs are too think & $\begin{array}{l}\text { 5. Acho que minhas pernas são finas demais e pouco } \\
\text { definidas na musculatura. }\end{array}$ \\
\hline $\begin{array}{l}\text { Appearance intolerance } \\
\text { 15. I am very shy about letting people see me } \\
\text { with my shirt off } \\
\text { 3. I wear loose clothing so that people cannot } \\
\text { see my body } \\
\text { 13. I feel like I have too much body fat } \\
\text { 9. I hate my body }\end{array}$ & $\begin{array}{l}\text { Intolerância à aparência } \\
\text { 15. Eu sou muito tímido quando se trata de deixar } \\
\text { as pessoas me verem sem camisa. } \\
\text { 3. Eu uso roupas bem soltas para que as pessoas não } \\
\text { possam ver meu corpo. } \\
\text { 13. Eu sinto que tenho muita gordura corporal e } \\
\text { poucos músculos. } \\
\text { 9. Eu odeio meu corpo }\end{array}$ \\
\hline $\begin{array}{l}\text { Functional impairment } \\
\text { 19. I pass up chances to meet new people } \\
\text { because of my workout schedule } \\
\text { 18. I feel depressed when I miss one or more } \\
\text { workout days } \\
\text { 17. I pass up social activities (e.g. watching } \\
\text { football games, eating dinner, going to see a } \\
\text { movie, etc.) with friends because of my workout } \\
\text { schedule } \\
\text { 16. I feel anxious when I miss one or more } \\
\text { workout days }\end{array}$ & $\begin{array}{l}\text { Comprometimento funcional } \\
\text { 19. Eu deixo passar as chances de conhecer novas } \\
\text { pessoas por causa do meu horário de atividade } \\
\text { física na academia. } \\
\text { 18. Sinto-me chateado ou irritado quando deixo de } \\
\text { ir um ou mais dias as atividades físicas na academia. } \\
\text { 17. Eu deixo de viver minhas atividades sociais (por } \\
\text { exemplo, assistir a jogos de futebol, jantar, assistir a } \\
\text { um filme, ir a shows, etc.) com amigos por causa da } \\
\text { minha agenda de exercícios. } \\
\text { 16. Sinto-me ansioso quando não vou um ou mais } \\
\text { dias para as atividades físicas na academia. }\end{array}$ \\
\hline
\end{tabular}

\section{Estudo 2: Análise da discriminação e representatividade dos itens da escala de dismorfia muscular.}

A fim de identificar a relação do domínio teórico e empírica do conteúdo dos itens da MDDI, tomou-se como orientação as propostas psicométricas e conceituais de Schlundt, Woodford e Brownlee (2000) e Zeeck, Welter, Alatas, Hildebrandt, Lahmann e Hartmann (2018) para a MDDI.

Com isso, verificou-se, a discriminação e representatividade dos itens, as quais, têm como base de avaliação, verificar sistematicamente, a relação teórica da escala e as situações especificadas nos itens e o quanto eles representam os aspectos esperados (cf. Formiga, Fleury, Fandiño \& Souza, 2016).

Na primeira avaliação apresentou uma maior especificidade na análise estatística destinado a avaliação da organização distributivas dos itens na escala MDDI. Para a realização desta análise tem-se a orientação dos pressupostos da Teoria Clássica dos Testes (TCT), estas se referem a necessidade de avaliar se os itens são capazes de discriminar as respostas das pessoas com magnitudes próximas, 
considerando os grupos com pontuações inferiores e superiores com relação ao construto medido (cf. Formiga, Fleury, Fandiño \& Souza, 2016).

Para atender a essa condição hipotético-metodológica (a discriminação dos itens das escalas), foi gerada uma pontuação total para a MDDI e, em seguida, calculou-se a sua mediana. Desta maneira, os respondentes que apresentaram pontuação abaixo da mediana foram classificados como sendo do grupo inferior (GI) e aqueles com pontuações acima foram definidos como do grupo superior (GS) (cf. Formiga, Fleury, Fandiño, \& Souza, 2016). Isto é, GI e GS, são os grupos de respondentes que se encontram nos extremos concordante e discordantes da escala.

Considerando-se cada item da MDDI, efetuou-se um teste $\mathrm{t}$ de Student para amostras independentes e compararam-se ambos os grupos observando a discriminação de cada item da escala quanto as pessoas com magnitudes próximas estatisticamente significativas.

É preciso salientar que, para a realização desses cálculos, uma amostra inicial de 30 sujeitos foi coletada, os quais, tinham as semelhantes características sociodemográficas da amostra total destinadas ao estudo.

Na Tabela 2, pode-se observar o poder discriminativo dos itens na MDDI, não somente os 13 itens foram significativos, quanto foram capazes de discriminar as pessoas com magnitudes próximas, estatisticamente significativas.

Tabela 2

Discriminação dos itens da escala MDDI

\begin{tabular}{|c|c|c|c|}
\hline Itens & Grupos & $\mathbf{t}$ & p-valor \\
\hline MDDI 1 & $\begin{array}{l}\text { gi } \\
\text { gs }\end{array}$ & $-6,35$ & 0,01 \\
\hline MDDI 2 & $\begin{array}{l}\text { gi } \\
\text { gs }\end{array}$ & $-7,25$ & 0,01 \\
\hline MDDI 3 & $\begin{array}{l}\text { gi } \\
\text { gs }\end{array}$ & $-5,90$ & 0,01 \\
\hline MDDI 4 & $\begin{array}{l}\text { gi } \\
\text { gs }\end{array}$ & $-4,04$ & 0,01 \\
\hline MDDI 5 & $\begin{array}{l}\text { gi } \\
\text { gs }\end{array}$ & $-6,87$ & 0,01 \\
\hline MDDI 6 & $\begin{array}{l}\text { gi } \\
\text { gs }\end{array}$ & $-5,74$ & 0,01 \\
\hline MDDI 7 & $\begin{array}{l}\text { gi } \\
\text { gs }\end{array}$ & $-4,75$ & 0,01 \\
\hline MDDI 8 & $\begin{array}{l}\text { gi } \\
\text { gs }\end{array}$ & $-3,91$ & 0,01 \\
\hline MDDI 9 & $\begin{array}{l}\text { gi } \\
\text { gs }\end{array}$ & $-4,44$ & 0,01 \\
\hline MDDI 10 & $\begin{array}{l}\text { gi } \\
\text { gs }\end{array}$ & $-5,07$ & 0,01 \\
\hline MDDI 11 & $\begin{array}{l}\text { gi } \\
\text { gs }\end{array}$ & $-9,52$ & 0,01 \\
\hline MDDI 12 & $\begin{array}{l}\text { gi } \\
\text { gs }\end{array}$ & $-5,91$ & 0,01 \\
\hline
\end{tabular}




\begin{tabular}{cccc} 
MDDI 13 & gi & & \\
gs & $-7,05$ & 0,01 \\
\hline Nota: ${ }^{*} \mathrm{p}<0,05 ; \mathrm{r}=$ correlação de Pearson; $\mathrm{t}=$ & teste de Student
\end{tabular}

Atento de que à análise da discriminação dos itens foi significativa, realizou-se a análise de representatividade de conteúdo itens-fator, a qual, é avaliada através do cálculo de correlação (r) de Pearson.

Na tabela 3, é possível observar as correlações de Pearson (r), o qual tem o objetivo de avaliar a relação dos itens da escala com a pontuação total da MDDI, condição a qual, espera-se que sejam significativos e tenham correlações acima de 0,50 (cf. Formiga, Fleury, Fandiño \& Souza, 2016).

0 motivo em considerar o limite mínimo deste escore correlacional para a avaliação da representatividade de conteúdo, deve-se ao fato de encontrar na literatura de que neste intervalo de interpretação, a possibilidade de que é possível afirmar uma aproximação empírica entre as variáveis, as quais, hipotetizadas teoricamente, é muito maior do que escores menores (cf. Figueiredo Filho, \& Silva Junior, 2010; Cohen, Swerdlik, \& Sturman, 2014).

A partir do cálculo de correlação de Pearson (r), na tabela 2, é possível observar a existência de uma relação itens-constructo, com todos eles se correlacionando positivamente e significativos, acima de 0,50 . Pode-se destacar que, de todos esses itens, representam a proposta da construção de uma escala MDDI.

Tabela 3

Representatividade de conteúdo

\begin{tabular}{ccc}
\hline Itens & MDDItotal & p-valor \\
& & \\
\hline MDDI 1 & 0,58 & 0,001 \\
MDDI 2 & 0,60 & 0,001 \\
MDDI 3 & 0,57 & 0,001 \\
MDDI 4 & 0,52 & 0,001 \\
MDDI 5 & 0,52 & 0,001 \\
MDDI 6 & 0,56 & 0,001 \\
MDDI 7 & 0,55 & 0,001 \\
MDDI 8 & 0,55 & 0,001 \\
MDDI 9 & 0,53 & 0,001 \\
MDDI 11 & 0,51 & 0,001 \\
MDDI 11 & 0,69 & 0,001 \\
MDDI 12 & 0,51 & 0,001 \\
MDDI 13 & 0,60 & 0,001 \\
\hline
\end{tabular}

\section{Estudo 3: Análise fatorial exploratória e de consistência interna da escala MDDI}

A partir dos resultados observados nas duas análises anteriores, realizou-se uma análise fatorial exploratória (AFE). Nesta etapa, participaram do estudo 170 respondentes, os quais praticantes de atividades físicas em academias.

Para a realização da AFE, foram estabelecidos os seguintes critérios (cf. Dancey \& Reidy, 2006): o método dos Componentes Principais (PC), deixando livre o número de fatores a que se pretende extrair, fixando a fatorialidade na rotação fatorial oblíqua (oblimin) e estabelecendo uma saturação de $\geq 0,30$ para a retenção dos fatores.

Para ter maior segurança na tomada de decisão na escolha dos fatores, adotaram-se outros critérios para a avaliação fatorial: (1) quantidade de valores próprios (eigenvalues) $\geq 1,00$ (Critério de Kaiser), (2) distribuição gráfica dos valores próprios, isto é, o gráfico de declividade, no qual, pode-se indicar, quanto referência, o ponto do corte a partir que limita a quantidade de fatores consideráveis para a estrutura fatorial (Critério de Cattell) e (3) análise paralela (O’Connor, 2000; Dancey, \& Reidy, 2006). 
A partir desses critérios, os resultados da AFE permitiram identificar a adequação da matriz de correlação: $\mathrm{KMO}=0,72$ e do Teste de Esfericidade de Bartlett, $\chi^{2} / \mathrm{gl}=680,23 / 78, p<0,001$. Na distribuição gráfica tendo como base os valores próprios (critério de Cattell), foram identificados quatro fatores na escala com valores próprios maiores do que 1,00 (ver Figura 1).

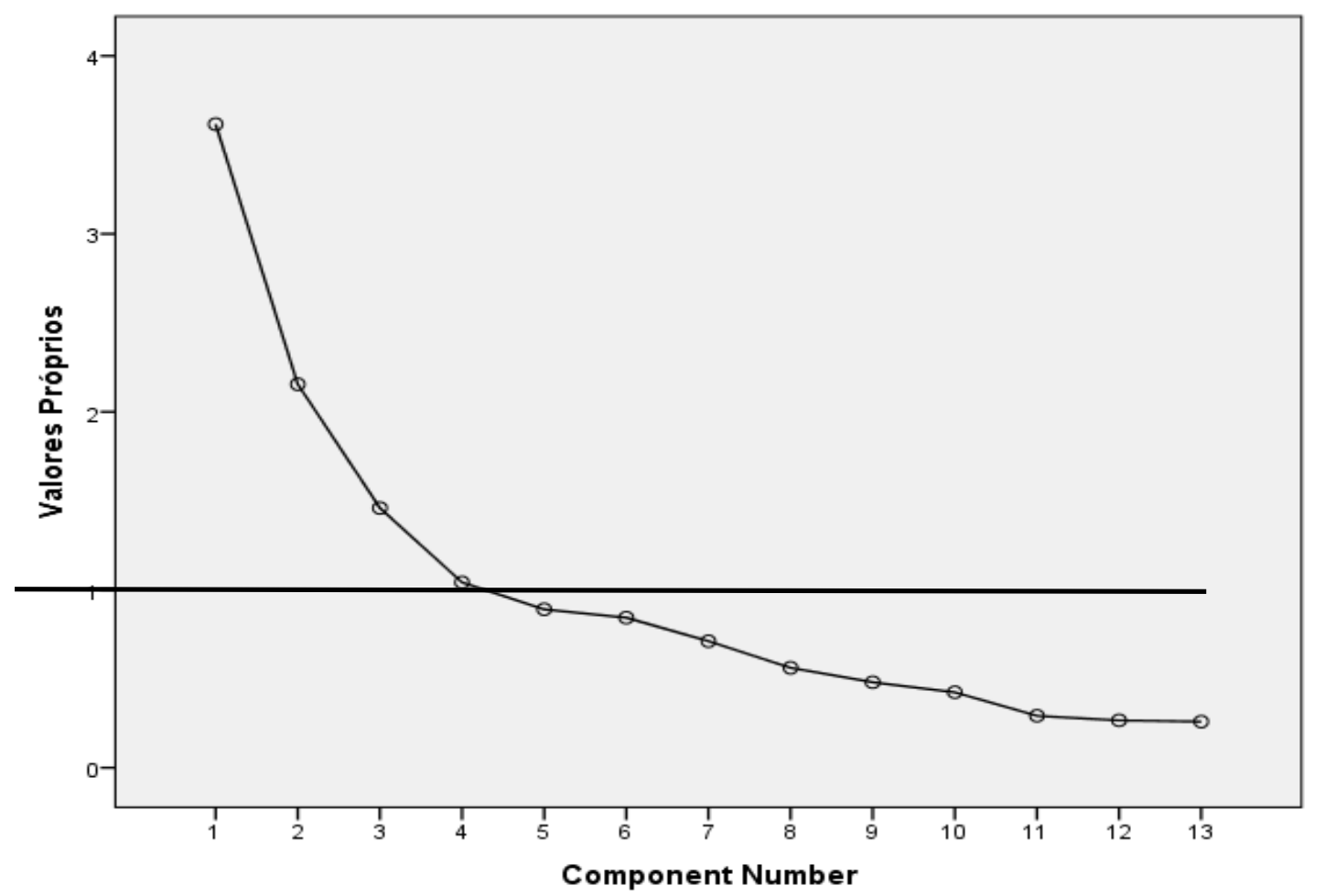

Figura 1. Diagrama de declividade do número de fatores plotados da MDDI

$\mathrm{Na}$ avaliação do critério de Kaiser (isto é, os valores próprios - Eigenvalues), também, identificou-se a existência de quatro fatores com valores próprios maiores que 1 (um), explicando conjuntamente $63,63 \%$ da variância total do fenômeno avaliado.

Para não deixar dúvidas referente a organização fatorial, pios, teoricamente, são estabelecidos três fatores para a MDDI, realizou-se o cálculo da análise paralela; para este cálculo, assumiu o mesmo parâmetro do banco de dados original (170 participantes e 13 variáveis-itens relativo à escala MDDI), tendo seus valores próprios gerados em 1.000 simulações aleatórias com os itens (Ver Tabela 4).

\section{Tabela 4}

Distribuição dos fatores da análise PC de acordo com o critério de Kaiser e análise paralela

\begin{tabular}{cc}
\hline Critério de Kaiser & Análise paralela \\
\hline $\mathbf{3 , 6 2}$ & $\mathbf{1 , 4 8}$ \\
$\mathbf{2 , 1 6}$ & $\mathbf{1 , 3 5}$ \\
$\mathbf{1 , 4 6}$ & $\mathbf{1 , 3 2}$ \\
1,04 & 1,18 \\
\hline
\end{tabular}

Na tabela 4 quando se compararam os escores dos valores próprios observados nos critérios de Kaiser e os da análise paralela, observou-se que, daqueles encontrados no critério de Kaiser, apenas três apresentaram valores superiores aos simulados (análise paralela); com isso, para a fatorialização da MDDI, a organização tri-fatorial é a mais recomendada para a realização da AFE.

Ainda que tais resultados revelem uma garantia para a distribuição dos fatores, consultaram-se juízes especializados (no conhecimento da estatística e avaliação psicológica) a fim de avaliar a decisão tomada pela autora; atento a interpretação do item-fator estabelecida juízes, o julgamento deles 
contribuiu para corroborar a decisão que se esperava: a tri-fatorialidade da escala.

A partir de tais critérios, procedeu-se a realização de uma análise fatorial do Componentes Principais (PC) com rotação oblíqua, eigenvalue (valor próprio) $\geq 1,00$ para definir o fator e saturação de $\geq 0,30$ para retenção dos itens. A uso desta técnica se mostrou bastante adequada, tendo os indicadores estatísticos (KMO = 0,72 e do Teste de Esfericidade de Bartlett, $\chi^{2} / \mathrm{gl}=680,23, p<0,001$ ) revelado a possibilidade de que a fatorilização seja realizada.

Desta maneira, os resultados desta extração fatorial, revelaram que a MDDI tem sua organização em três fatores, o qual, segue em direção semelhante ao resultado encontrado nas análises estatísticas primárias quando na tomada de decisão - valores próprios (eigenvalues) iguais ou superiores a 1 (Critério de Kaiser), distribuição gráfica dos valores próprios (Critério de Cattell) e a análise paralela - as quais, revelaram tanto uma organização lógicos relativa a associação item-fator, quanto, os escores fatoriais estiveram acima do padrão exigido $(\geq 0,50)$ (ver tabela 5).

Tabela 5

Análise fatorial dos principais componentes da MDDI

\begin{tabular}{|c|c|c|c|c|}
\hline Itens & DT & IA & CF & $\mathbf{h}^{2}$ \\
\hline MDDI 1 & 0,82 & --- & --- & 0,38 \\
\hline MDDI 2 & 0,73 & --- & --- & 0,68 \\
\hline MDDI 3 & 0,67 & --- & --- & 0,57 \\
\hline MDDI 4 & 0,63 & --- & --- & 0,45 \\
\hline MDDI 5 & 0,53 & --- & --- & 0,48 \\
\hline MDDI 6 & --- & 0,77 & --- & 0,50 \\
\hline MDDI 7 & --- & 0,75 & --- & 0,56 \\
\hline MDDI 8 & --- & 0,73 & --- & 0,63 \\
\hline MDDI 9 & --- & 0,69 & --- & 0,54 \\
\hline MDDI 10 & --- & --- & 0,82 & 0,61 \\
\hline MDDI 11 & --- & --- & 0,78 & 0,56 \\
\hline MDDI 12 & --- & --- & 0,77 & 0,69 \\
\hline MDDI 13 & --- & --- & 0,70 & 0,60 \\
\hline $\begin{array}{l}\text { Número total de } \\
\text { itens }\end{array}$ & 5 & 4 & 4 & 13 \\
\hline Valores próprios & 3,16 & 2,15 & 1,46 & 6,77 \\
\hline Variância explicada & $27,82 \%$ & $16,58 \%$ & $11,22 \%$ & $55,62 \%$ \\
\hline Alfa de Cronbach & $0,72^{*}$ & $0,72^{*}$ & $0,77^{*}$ & $0,77^{*}$ \\
\hline $\begin{array}{c}\text { ICC } \\
(95 \% \text { IC) }\end{array}$ & $\begin{array}{c}0,72^{*} \\
(0,70-0,78)\end{array}$ & $\begin{array}{c}0,72^{*} \\
(0,70-0,78)\end{array}$ & $\begin{array}{c}0,77^{*} \\
(0,70-0,82)\end{array}$ & $\begin{array}{c}0,77^{*} \\
(0,71-0,82)\end{array}$ \\
\hline $\begin{array}{l}\text { Escore médio do } \\
\text { construto }\end{array}$ & 8,35 & 5,10 & 3,44 & 17,92 \\
\hline
\end{tabular}

Nota: ${ }^{*} \mathrm{p} \geq 0,001 ; \mathrm{DT}=$ Desejo por tamanho do corpo; IA = Intolerância á Aparência; $\mathrm{CF}=$ Comprometimento Funcional 
Na tabela 5, é possível avaliar e a distribuição de todos os fatores da MDDI; o primeiro fator, definido como Desejo por tamanho do corpo (refere-se aos pensamentos que o respondente tem em relação a ser menor, menos musculoso e mais fraco que o desejado, tendo explicito interesse em aumentar o tamanho e a força), explicou conjuntamente $27,82 \%$ da variância do fator, composto cinco itens (1. A minha vontade é de ficar mais forte, bem malhado e definido; 2. Acho que não tenho músculos o bastante, isto é, não sou 'fortão'; 3. Acho que meu peitoral não tem músculos tão bem definidos, do tipo malhadão; 4. Minha vontade é que meus braços tivessem músculos mais definidos e malhados; 5. Acho que minhas pernas são finas demais e pouco definidas na musculatura). Destaca-se também, que este fator revelou alfa e ICC acima de 0,70; este último indicador, refere-se a qualidade da consistência da medida destinada a uma administração futura do MDDI numa amostra com características semelhantes.

0 segundo fator, categorizado como Intolerância à Aparência (diz respeito as crenças negativas sobre o corpo e a ansiedade causada pela aparência ou prevenção da exposição corporal, condicionando o respondente a procurar vestir roupas largas na praia ou crer que o corpo é feio e desagradável), explicou 16,58\% e é formado por 4 itens (6. Eu sou muito tímido quando se trata de deixar as pessoas me verem sem camisa; 7. Eu uso roupas bem soltas para que as pessoas não possam ver meu corpo; 8. Eu sinto que tenho muita gordura corporal e poucos músculos; 9. Eu odeio meu corpo). Neste fator, observou-se, também, uma alfa acima de 0,70 , bem como o ICC.

Por fim, o terceiro fator, categorizado como Comprometimento Funcional (tem sua definição centrada nos comportamentos relativos à manutenção de rotinas de exercícios, capaz de influenciar nas emoções de forma negativa quando o respondente se desvia das rotinas de exercícios ou de suas atividades interpessoais, exclusivamente, para se preocupar com o seu corpo), explicou 11,22\%, o qual, é formado por 4 itens (10. Eu deixo passar as chances de conhecer novas pessoas por causa do meu horário de atividade física na academia; 11. Sinto-me chateado ou irritado quando deixo de ir um ou mais dias as atividades físicas na academia; 12. Eu deixo de viver minhas atividades sociais (por exemplo, assistir a jogos de futebol, jantar, assistir a um filme, ir a shows, etc) com amigos por causa da minha agenda de exercícios. 13. Sinto-me ansioso quando não vou um ou mais dias para as atividades físicas na academia). Neste fator, o alfa também, foi acima 0,70 e o ICC encontra-se no limite psicométricos aceito pela literatura.

Na mesma tabela 5, apresentou a distribuição dos itens por fatores, sua saturação (carga fatorial) e comunalidade, bem como, os indicadores de consistência interna (Alfa de Cronbach), variância explicada pelo fator e a correlação intra-classe. Todos os indicadores estiveram dentro do padrão psicométrico exigido (Hair et al., 2005; Pasquali, 2011), o que garante a qualidade fatorial da escala MDDI para a versão em brasileiros.

No que se refere aos escores médios de cada fator da medida em questão, pode-se observar na tabela 5 que, hierarquicamente, o fator DT foi o que apresentou maior média, seguido de IA e, posteriormente, de CF. Pode-se destacar, que nesta amostra, o fator DT é mais prevalente.

Com a escala MDDI garantindo a sua tri-fatorialidade em frequentadores de academia, procurou-se verificar a relação entre os fatores da MDDI; com base numa correlação de Pearson (r), observou-se que todos os fatores estiveram relacionados, positivamente entre eles. Porém, chama-se atenção que, nas relações DF-IA e IA-CF, foram observadas correlações abaixo de 0,30, pois, mesmo sendo significativas, é preciso ter alguma parcimônia ao afirmar, de forma convicta, a influência nesta relação.

Tabela 6

Escores intercorrelacionais entre os fatores da MDDI.

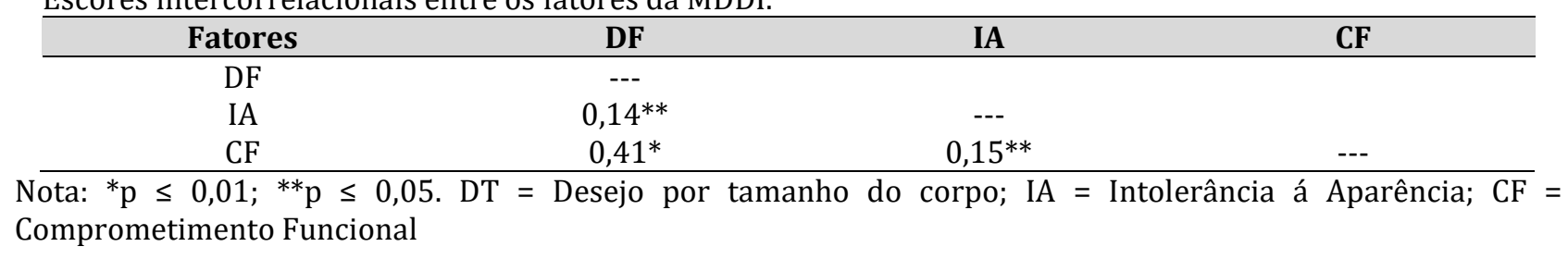


Um resultado adicional foi realizado, a partir de um teste $t$ para amostras pareadas; neste, pretendeu avaliar as diferenças nos escores médios entre os fatores da MDDI. Na tabela 7, observou-se que o fator DT apresentou escore médio superior aos demais fatores (IA e CF), enquanto no pareamento IA-CF, a IA apresentou maior escore. Considerando estes resultados, é possível estabelecer uma direção hierárquica dos fatores das MDDI para tais participantes: DF > IA > CF.

Tabela 7

Pareamento entre os escores médios nos fatores do MDDI.

\begin{tabular}{|c|c|c|c|c|c|c|c|}
\hline \multirow{2}{*}{\multicolumn{2}{|c|}{ Pares de variáveis }} & \multirow{2}{*}{ Média } & \multirow{2}{*}{ d.p. } & \multicolumn{2}{|c|}{ IC $95 \%$} & \multirow{2}{*}{$\mathbf{t}$} & \multirow{2}{*}{$\mathbf{p} \leq$} \\
\hline & & & & Baixo & Alto & & \\
\hline \multirow{2}{*}{ Par 1} & DT & 8,35 & 4,41 & \multirow{2}{*}{2,13} & \multirow{2}{*}{4,08} & \multirow{2}{*}{7,80} & \multirow{2}{*}{0,01} \\
\hline & IA & 5,09 & 3,65 & & & & \\
\hline \multirow{2}{*}{ Par 2} & DT & 8,35 & 4,41 & \multirow{2}{*}{4,24} & \multirow{2}{*}{5,59} & \multirow[t]{2}{*}{14,44} & \multirow[t]{2}{*}{0,01} \\
\hline & $\mathrm{CF}$ & 3,43 & 3,61 & & & & \\
\hline \multirow{2}{*}{ Par 3} & IA & 5,09 & 3,65 & \multirow{2}{*}{0,93} & \multirow{2}{*}{2,39} & \multirow[t]{2}{*}{4,47} & \multirow[t]{2}{*}{0,01} \\
\hline & $\mathrm{CF}$ & 3,43 & 3,61 & & & & \\
\hline
\end{tabular}

A partir desses resultados, optou-se em avaliar a associações dos fatores (DF, IA e CF) das MDDI e as variáveis sociodemográficas. Realizou-se uma Anova One-way, considerando o fator DF em função do sexo, nível etário e estado civil observaram-se resultados significativos no efeito de interação na variável idade vs estado civil. Os respondentes de 34 e 56 anos e solteiros apresentaram escores maiores $\left[F(2,170=2,80, p<0,05), \mathrm{OP}=0,76, \eta^{2}=0,07\right]$, em relação aos demais.

No que se refere a IA, houve um resultado significativo apenas efeito direto na variável sexo $[F$ $\left.(2,170=4,22, p<0,05), \mathrm{OP}=0,53 \eta^{2}=0,03\right]$, tendo as mulheres apresentado escores maiores do que os homens. Por fim, quanto ao $\mathrm{CF}$, observou-se significância no efeito direto da variável sexo $[F(2,170$ $\left.=5,68, p<0,05), \mathrm{OP}=0,66, \eta^{2}=0,04\right]$ e no efeito de interação da variável sexo vs estado civil $[F(2,170$ $=6,27, p<0,05$ ), OP $\left.=0,89, \eta^{2}=0,08\right]$. No primeiro efeito, os homens pontuaram mais alto do que as mulheres e no efeito de interação, os homens e viúvos tiveram escores maiores do que os demais.

Verificou-se também, o quanto o tempo dedicado em horas de atividade física na academia influenciaria o DF, IA e CF. A partir da Anova one-way, observou-se que não houve resultado significativo; mas, é necessário chamar à atenção, que mesmo não sendo significativos a variação.

A partir desses resultados, optou-se em verificar a validade convergente da medida MDDI, associada ao construto da autoestima. Este construo é avaliado em duas dimensões: autoestima negativa e positiva. Com base numa correlação e Pearson, observou-se a existência uma relação positiva entre DT e autoestima negativa e positiva (respectivamente, $r=0,30,0,28$ ); a IA, apenas com a autoestima positiva $(\mathrm{r}=26)$ e, $\mathrm{CF}$ correlacionando com autoestima negativa $(\mathrm{r}=0,22)$ e positiva $(\mathrm{r}=$ $0,16)$.

\section{DISCUSSÃO}

Em termos gerais, este artigo teve como objetivo central traduzir, adaptar e validar, a partir dos indicadores psicométricos, previamente estabelecidos, a escala desordem da dismorfia muscular em frequentadores de academia, com uma amostra de sujeitos na cidade de Natal, RN.

Tomou-se como referência para à avaliação dos critérios psicométricos, os estudos desenvolvidos por Schlundt, Woodford e Brownlee (2000), Hildebrandt, Langenbucherb e Schlundtc (2004) e Zeeck, Welter, Alatas, Hildebrandt, Lahmann e Hartmann (2018), os quais, efetuaram-se análises estatísticas semelhantes as que foram realizadas no presente estudo. Neste, outros cálculos, não efetuados pelos autores supracitados com base na TCT (por exemplo, análise discriminativa dos itens e representatividade do conteúdo) também, foram realizados, condição, a qual, acredita-se ser um indicador que contribuiria bastante quanto evidência analítica para a verificação associativa itemfator da MDDI e que acrescentaria mais informação empíricas para a qualidade da medida em questão. 
Com isso, no desenvolvimento dos estudos avaliativos da MDDI, não apenas buscou-se oferecer maior segurança na análise da organização fatorial desse instrumento, mas, também, oferecer mais elementos preliminar à consistência da referida medida.

Desta maneira, avaliaram-se tanto a condição de discriminação dos itens das escalas quanto a sua representatividade referente ao conceito e sentido do construto abordado. A partir destes cálculos, a fim de avaliar de forma inicial, a qualidade discriminativa da medida do item, pois, acredita-se que reter o item para o construto quando diretamente se efetua a análise fatorial, seja devido a uma crença pessoal do próprio pesquisador ou apenas por seguir o generalismo dessas análises, justificando motivos de que 'se todos fazem assim, também, pode-se fazer o mesmo', não é uma atitude suficiente e de força psicométrica. Viu-se a necessidade de avaliar se o respondente é capaz de indicar a partir do grau de concordância a sua compreensão do item (isto é, se o sujeito respondia para mais ou para menos nos extremos da escala de forma adequada em relação ao item) (cf. Cook \& Beckman, 2006; Pasquali, 2011).

Realizado o cálculo, especificamente, o teste t de Student, confirmou-se que os participantes conseguiram compreender o sentido e especificidade dos itens; na mesma direção foi verificando a representatividade dos itens, observando que os escores correlacionais foram fortes, positivos e significativos. Isto é, para todos os itens da MDDI, os respondentes indicaram compreender as questões associadas ao construto apresentado.

Certo de que os participantes forneciam sentido e direção correta na verificação do construto, foi que se efetuou a análise fatorial, a qual, apresentou um organização fatorial com três dimensões, bastante semelhantes aos resultados encontrados por Schlundt, Woodford e Brownlee (2000), Hildebrandt, Langenbucherb e Schlundtc (2004) e Zeeck, Welter, Alatas, Hildebrandt, Lahmann e Hartmann (2018). Os resultados observados com à amostra brasileira diferenciou-se nos escores fatoriais, tendo estes, sido muito maior do que os observados pelos autores supracitados (todos acima de 0,50).

Para garantir a fatorialização da MDDI, realizou-se tanto o alfa de Cronbach, o qual apresentou alfa acima de 0,70, seja na pontuação total, seja para cada dimensão da MDDI. Além desse indicador, foi calculado também, a correlação intra-classe (o ICC), o qual, tem como orientação psicométrica, o uso da MDDI no futuro, com novas amostras que apresentem semelhantes características com à amostra coletada. Com isso, de forma geral, pode-se afirmar que a escala MDDI numa amostra brasileira se organiza em três dimensões, de acordo com o que foi proposto pelos autores originais desta escala.

Não somente foram observados indicadores psicométricos confiáveis, os quais, garantiram a qualidade da mensuração da escala de desordem da dismorfia muscular em pessoas que frequentam academia; bem como, pode-se também destacar que, para esta amostra, a partir desses resultados, o MDDI é capaz de avaliar o seu estado psicológico do respondente, com base na sua autoavaliação relativa ao sua imagem corporal-distrófica.

Considerando que a medida MDDI é consistente, destacou-se, numa avaliação de convergência com a autoestima (positiva e negativa) que estes construtos se associam. Mas, apesar das correlações serem positivas, os escores não estiveram muito consistentes ao padrão de convergência, o qual, é exigido um indicador ' $r$ ' de Pearson acima de 0,50 .

Com isso, os resultados correlacionais contribuem para pressupor que existe uma relação direta entre a desordem da dismorfia e a autoestima, mas, que esta associação precisa ser atribuída com parcimônia devido ao escore estar entre baixa e moderada correlação. Ainda assim, é possível atribuir que, aquele sujeito que pontuar alto na escala MDDI, provavelmente, pontuará alto na escala de autoestima.

Estes achados podem ser útil quanto alerta no que se refere ao exagero nos exercícios, pois, o sujeito que busca tal atitude distrófica em maior ou menor grau, geralmente, está associado a todo tipo de exercício em acadêmica, a qual, propõem esta condição; bem como, chamar atenção para considerar que poderá ser tratar de um problema muito, pois, as pessoas se concentram na distrofia em termos de uma desordem, a partir desses resultados, ela está associada ao sistema psicológico da autoestima e, provavelmente, gerar problemas mais psiquicamente mais sérios, por exemplo: atitudes anoréxicas e bulímicas. 
De forma geral, esses resultados sugerem maior atenção na condição motivadora e estética do estímulo as atividades físicas na academia, tanto no que se refere a estrutura e organização do exercício a determinadas pessoas, quanto a funcionalidade na demanda e cumprimentos das atividades na academia, afinal, o comedimento é sinônimo de possível saúde psíquica e física, bem como, de relações interpessoais.

\section{CONSIDERAÇÕES FINAIS}

De forma geral, a partir desses resultados, acredita-se que tenha alcançado os objetivos, os quais: verificar a validade de conteúdo e fatorial da The Muscle Dysmorphic Disorder Inventory - MDDI (inventário sobre dismorfia muscular) em frequentadores de academia.

Assim, os resultados apresentados pretenderam contribuir para a explicação de uma área da psicologia do consumo e a autoavaliação da imagem da função e estrutura corporal frente ao ajustamento psicológico dos praticantes de atividades físicas na cidade de Natal-RN.

Mesmo com resultados bastante significativos, os quais, corroboram a proposta original dos autores das MDDI, tanto em termos teóricos quanto de organizacional fatorial; ainda assim, é preciso destacar alguns limites no estudo: seria útil um estudo mais amplo, contemplando tanto as características das mesmas pessoas quanto outras mais, especialmente, as praticantes de esportes de baixo, médio e alto nível; outra proposta interessante seria associar essa medida a construto que avaliam transtorno alimentar, bem como, transtorno emocional; não menos importante, poderia desenvolver um estudo que avaliasse pessoas, longitudinalmente, na prática de atividades na acadêmica, bem como, associasse a variáveis psicológicas do bem-estar subjetivo, autoestima, autoimagem corporal.

Com isso, seria muito importante em estudos futuros contemplar as dimensões locais, específicas ou exclusivas da orientação de cada uma cultura, com o objetivo de comparar os construtos estudados aqui para outro espaço geopolítico e social, já que se acredita que os respondentes poderão mudar de concepção e até percepção, quando na mudança do contexto sócio organizacional, condição essa que sugere novas aplicações desta medida para avaliar a manutenção do construto no espaço e prática do exercício físico.

De forma geral, a MDDI não se trata, somente, de uma medida garantida, espera-se que seja utilizada para avaliar a qualidade de vida social e mental do praticante de atividade física, bem como, possibilidade desenvolva possibilidades de intervenção psicossocial quando se pretender investir no desenvolvimento e manutenção emocional das pessoas quando na iniciação de atividades físicas, seja por motivo de saúde, seja devido a busca de uma estética corporal.

\section{References}

Armstrong, S. J., \& Overton, T.S. (1997). Estimating non-responses bias in mailed surveys. Journal of Marketing Research, 13, 396 - 402.

Associação Americana de Psiquiatria. (2013). Manual Diagnóstico e Estatístico de Transtornos Mentais (5 ed.). Arlington, VA: American Psychiatric Publishing.

Associação Nacional de Pesquisa e Pós-Graduação em Psicologia - ANPEPP. (2000). Contribuições para a discussão das Resoluções CNS n0 196/96 e CFP N0 016/2000. Recuperado em 07 de Agosto de 2012, da: HTTP://www.anpepp.org.br/XIISimposio/Rel_ComissãoEticasobre_Res_CNS_e_CFP.pdf.

Assunção, S. S. M. (2002). Dismorfia muscular. Rev. Bras. Psiquiatr. 24(supl III) 80-4.

Azevedo, A.P., Ferreira, A.C. Da Silva P.P., Caminha, I.O., Freitas, C.M. (2012). Dismorfia muscular: A busca pelo corpo hiper musculoso. Motricidade, 8(1), 53-66.

Branden, N. (2000). Autoestima e seus seis pilares. 6⿳a ed. São Paulo: Saraiva.

Cohen, R. J., Swerdlik, M. E., \& Sturman, E. D. (2014). Testagem e avaliação psicológica: introdução a testes e medidas (8a ed). Porto Alegre, RS: AMGH. 
Conselho Nacional de Saúde - CNS (2012). Diretrizes e Normas Regulamentadoras de Pesquisas Envolvendo Seres Humanos. Recuperado em 07 de Agosto de 2012, da http://conselho.saude.gov.br/resolucoes/reso_96.htm

Dancey, C. P., \& Reidy, J. (2006). Estatística sem matemática para psicologia. Porto Alegre: Artmed.

Debord, Guy. (1997). A Sociedade do Espetáculo. Rio de Janeiro: Contraponto,

Faul F., Erdfelder E., \& Buchner A., Lang A.-G. (2009). Statistical power analyses using G*Power 3.1: tests for correlation and regression analyses. Behavior Research Methods, 41, 1149-1160

Figueiredo Filho, D., \& Silva Junior, J. (2010). Desvendando os Mistérios do Coeficiente de Correlação $\begin{array}{lllll}\text { de Pearson } & \text { (r). Revista Hoje, 18(1). }\end{array}$ de https://periodicos.ufpe.br/revistas/politicahoje/article/view/3852

Filho, C. A. A. C. (2000) O discurso do profissional de ginástica de academia no Rio de Janeiro. Movimento, ano VI, n. 12.

Forbes, G. B., Jung, J., Vaamonde, J. D., Omar, A., Paris, L., \& Formiga, N. S. (2012). Body dissatisfaction and disordered eating in three cultures: Argentina, Brazil, and the U.S. Sex Roles, 66(9-10),677694. doi: 10.1007/ s11199-011-0105-3

Formiga, N., Fleury, L. F. O., Fandiño, A. M., \& Souza, M. A., (2016). Evidência Empírica de uma medida da Anomia Organizacional em trabalhadores brasileiros. Revista de Psicologia, 18(1), 43-59.

Fortes, L. de S., Paes, S. Tavares, R. Júnior, Dilson Borges, A., S. de Sousa, \& Ferreira, M. E. C. (2017). Busca pela muscularidade, humor e transtornos alimentares em atletas do sexo masculino. Revista Brasileira de Medicina do Esporte, 23(1), 37-41. https://dx.doi.org/10.1590/1517869220172301136184.

Giacomoni, C. H. (2002). Bem-estar subjetivo infantil: conceito de felicidade e construção de instrumentos para avaliação. Tese de Doutorado Não-Publicada, Programa de Pós-Graduação em Psicologia do Desenvolvimento. Universidade Federal do Rio Grande do Sul, Porto Alegre.

Gonçalves, A. S. (2014). Corpos modidificados ao extremo: O eu, o outro e a sociedade. Tese (Doutorado). Universidade de Brasília, UNB.

Hildebrandt T, Alfano L, Langenbucher JW. (2010). Body image disturbance in 1000 male appearance and performance enhancing drug users. Journal of Psychiatric Research, 44, 841-846. https://doi.org/10.1016/j. jpsychires.2010.01.001

Hutz, C. (2000). Adaptação brasileira da Escala de Autoestima de Rosenberg. Curso de Pós-Graduação em Psicologia, Universidade Federal do Rio Grande do Sul, Porto Alegre, Mimeo

Jesus, R. S.; Lopes, D. P.; Andrade, F. J.; Albieri, G. A. R.; Almeida, G. A.; Rocha, T. A. B. (2016). A influência dos padrões de beleza impostos pela mídia. Universidade São Judas Tadeu. São Paulo. Disponível em: conicsemesp.org.br/anais/files/2016/trabalho-1000022371.pdf.

Kelloway, E. K. (1998). Using LISREL for structural equation modeling: A researcher's guide. Sage.

Ledesma, R. D. \& Valero-Mora, P. (2007). Determining the number of factors to retain in EFA: An easyto-use computer program for carrying out parallel analysis. Practical Assessment, Research and Evaluation, 12(1), 1-11.

Lipovetsky, G. (1989). O Império do Efêmero: a moda e seu destino nas sociedades modernas. São Paulo: Companhia das Letras.

Lipovetsky, Gilles (2007). A felicidade paradoxal: ensaios sobre a sociedade de hiperconsumo. São Paulo: Companhia das Letras.

Marques, F. A., Legal, E., J., \& Höfelmann, D. A. (2012). Insatisfação corporal e transtornos mentais comuns em adolescentes. Revista Paulista de Pediatria, 30(4), 553-561. https://doi.org/10.1590/S0103-05822012000400014

Mendes, M. I. B. S., \& Nóbrega, T. P. (2004). Corpo, natureza e cultura: contribuições para a educação. Revista Brasileira de Educação, (27), 125-137. https://doi.org/10.1590/S141324782004000300009

Miles, J., \& Shevlin, M. (2001). Applying regression \& correlation. A guide for students and researchers. London: Sage Publications.

Miot H. A. (2017). Avaliação da normalidade dos dados em estudos clínicos e experimentais. Jornal vascular brasileiro, 16(2), 88-91. doi:10.1590/1677-5449.041117 
Moré, C., et al. (2008) Psicologia, saúde e trabalho: A ética na escuta psicológica: Atendimento inicial e a fila de espera. In: PLONER, KS., et al., org. Ética e paradigmas na psicologia social [online]. Rio de Janeiro: Centro Edelstein de Pesquisas Sociais. p. 253-272. ISBN: 978-85- 99662-85-4. Available from SciELO Books.

Motter, A. M., Almeida, S. \& Bellini, M. (2017). Incidência de vigorexia em praticantes de musculação. Do corpo: ciência e artes, 7(1), 117-127. Disponível em: http://www.ucs.br/etc/revistas/index.php/docorpo/article/view/5958/3200

Nascimento, D. C., Tibana R. A., Ferreira, G. M., \& Prestes, J. (2014) Testes de normalidade em análises estatísticas: uma orientação para praticantes em ciências da saúde e atividade física. Revista Mackenzie de Educação Física e Esporte, 14(2), 73-77.

Neumark-Sztainer D., Paxton, S. J., Hannan, P. J., Haines, J., \& Story, M. (2006). Does body satisfaction matter? Five-year longitudinal associations between body satisfaction and health behaviors in adolescent females and males. Journal Adolescent Health, 39, 244-51.

Olivardia, R. (2001). Mirror, Mirror on the Wall, Who's the Largest of Them All? The Features and Phenomenology of Muscle Dysmorphia. Harvard Review of Psychiatry, 9(5), 254-259. Doi: https://doi.org/10.1080/hrp.9.5.254.259

Petroski, E. L., Pelegrini, A., Glaner, M. F. (2012) Motivos e prevalência de insatisfação com a imagem corporal em adolescentes. Núcleo de Pesquisa em Cineantropometria e Desempenho Humano, Programa de Pós-graduação em Educação Física, Centro de Desportos, Universidade Federal de Santa Catarina.

Podsakoff, P. M., MacKenzie, S. B., Lee, J-Y, \& Podsakoff, N. P. (2003). Common method biases in behavioral research: a critical review of the literature and recommended remedies. Journal of Applied Psychology, 88(5), 879-903.

Pope, H.G., Gruber, A.J., Choi, P., Olivardia, R., Phillips, K.A. (1997). Muscle dysmorphia: an underrecognized form of body dysmorphic disorder. Psychosomatics, 38, 548-57.

Rosenberg, M. (1965). Society and the adolescent self-image. Princeton: PrincetonUniversity Press.

Rosenberg, M. (1973). La autoimagen del adolescente y la sociedad. Buenos Aires: Paidos. (Obra original publicada 1965).

Santos, A. R. M. Silva. Silva, E. A. P. C., Moura, P. V., et al. (2013) A busca pela beleza corporal na feminilidade e masculinidade. Revista Brasileira de Ciência e Movimento, 21(2), 135-142.

Schlundt, D. G., Woodford, H., \& Brownlee, A. (2000). Muscle dysmorphia in male weightlifters: Psychological characteristics and practices. Unpublished manuscrip

Severiano, M. de F. V. Rêgo, Montefusco, M. O. do, \& Real, É. V. (2010). O corpo idealizado de consumo: paradoxos da hipermodernidade. Revista Mal Estar e Subjetividade, 10(1), 137-165. Recuperado em 13 de julho de 2020, de http://pepsic.bvsalud.org/scielo.php?script=sci_arttext\&pid=S151861482010000100007\&lng=pt\&tlng=pt.

Tabachnick, B. G., \& Fidell, L. S. (2001). Using multivariate statistics. Needham Heights, MA: Allyn \& Bacon.

Tolentino, T. M., Maia, M. de F. de M., Formiga, N. S., Sousa, B. V. de O., \& Melo, G. F. (2016). Modelagem estrutural fatorial e consistência interna da escala de autoestima de Rosenberg em adolescentes brasileiros. Revista de Psicologia, 6(2), 40-49. Disponível em: http://www.periodicos.ufc.br/psicologiaufc/article/view/2580

Utiyama, A. H. B. (2011). Vigorexia: conceitos e problematização. Universidade Estadual Paulista. Rio Claro.

Disponível

em:

https://repositorio.unesp.br/bitstream/handle/11449/.../utiyama_ahb_tcc_rcla.pdf?...1

Vasconcelos, J. E. L. (2013). Vigorexia: quando a busca por um corpo musculoso se torna patológica. Revista de Educação Física- UNIFAFIBE, São Paulo, 91-97.

Wade, T. J. (2000). Evolutionary Theory and Self-Perception: Sex Differences in Body Esteem Predictors of Self-Perceived Physical and Sexual Attractiveness and Self-Esteem. International Journal of Psychology, 35, 36-45. http://dx.doi.org/10.1080/002075900399501.

Zeeck, A., Welter, V., Alatas, H., Hildebrandt, T., Lahmann, C., \& Hartmann, A. (2018). Muscle Dysmorphic Disorder Inventory (MDDI): Validation of a German version with a focus on gender. PLoS ONE, 13(11), Article e0207535. https://doi.org/10.1371/journal.pone.0207535 